\title{
RBEP
}

\section{Aprender no ensino superior: relações com a predisposição dos estudantes para o envolvimento na aprendizagem ao longo da vida}

Rita Barros

Angélica Reis Monteiro

J. António Marques Moreira

http://dx.doi.org/10.1590/S2176-6681/305811495

\section{Resumo}

Pretende analisar a relação entre a aprendizagem, com base na apropriação que os estudantes fazem das diferentes formas de aprender e de estudar, e a disposição que apresentam para se envolver em atividades de aprendizagem ao longo da vida (ALV). O rationale teórico inscreve-se na Teoria de Biggs (1987), no que concerne às abordagens de aprendizagem por parte do estudante, e no princípio orientador da ALV. Procedemos a um estudo comparativo-correlacional, com uma amostra constituída por 163 estudantes do ensino superior. As abordagens de aprendizagem foram avaliadas por meio da tradução luso-portuguesa da validação brasileira da escala Revised Two-factor Study Process Questionnaire (R-SPQ-2F). Os resultados obtidos permitem-nos associar a abordagem profunda a algumas características do aprendiz ao longo da vida.

Palavras-chave: ensino superior; estilos de aprendizagem; aprendizagem ao longo da vida. 


\section{Abstract \\ Learning in higher education: relations with the predisposition of students to be involved in lifelong learning}

The current investigation proposes to analyze the relationship between learning, based on the appropriation that students make of the different ways of learning and studying, and their willingness to be involved in lifelong learning $(L L)$ activities. The theoretical rationale is inscribed in the Biggs' Theory (1987), concerning the student's approach to learning, and under the guiding principle of LL. We present a correlational study, with a sample of 163 higher education students. The approaches to learning were supported by the Revised Two-factor Study Process Questionnaire (R-SPQ-2F). The results obtained allow us to associate the deep approach to some characteristics of learners throughout their life.

Keywords: higher education; learning styles; lifelong learning.

\section{Introdução}

Diversos autores da área da educação, mais especificamente os que se centram na educação de jovens e adultos, procuram compreender os processos de estudo e as concepções de aprendizagem desses estudantes (Biggs, 1987, 1993; Entwistle; Ramsden, 1983).

Biggs, Kember e Leung (2001), sustentados no modelo PresságioProcesso-Produto (3P), desenvolveram uma versão mais simplificada do instrumento Student Process Questionnaire (Biggs, 1987) para a compreensão dos processos de estudo e abordagens à aprendizagem, o Revised Two-factor Study Process Questionnaire (R-SPQ-2F).

Esse instrumento avalia a relação entre as características dos estudantes e do contexto de ensino, a abordagem que estes fazem das tarefas de aprendizagem e os seus resultados.

Procuramos, neste artigo, relacionar as diferentes abordagens à aprendizagem de estudantes de uma escola superior de educação e de uma escola superior de saúde com a disposição que apresentam para se envolver em atividades de aprendizagem ao longo da vida (ALV).

A aprendizagem ao longo da vida, entendida como um paradigma que emerge no contexto da sociedade de conhecimento e informação, oferece a possibilidade de se perspectivar de forma diferente os fenômenos educativos e facilita a emergência de um quadro de pensamento que valoriza as aprendizagens que as pessoas realizam nos domínios pessoais, sociais e profissionais (Pires, 2007).

Ao traduzir-se simultaneamente em agenda política e campo de pesquisa, a aprendizagem ao longo da vida requer uma reflexão centrada nessa articulação. Os contributos da investigação nessa matéria, cujo 
impacto recai nos processos políticos e nas práticas de aprendizagem, devem oferecer uma análise crítica da sua contextualização social, bem como possíveis projeções do que a aprendizagem ao longo da vida poderá vir a ser (Olesen, 2006), considerando que o futuro desta encerra ainda indefinições e questionamentos importantes (Jarvis, 2010). Preece (2011) aponta a redução do hiato entre os discursos e as políticas como o maior desafio da Sexta Conferência Internacional de Educação de Adultos Confintea VI (Conferência..., 2009), ao mesmo tempo em que sublinha a necessidade de uma aposta séria em termos de investigação (Unesco, 2010).

O estudo que apresentamos, ao traduzir-se numa investigação em um contexto particular, apresenta-se como um contributo com o objetivo de colmatar essa necessidade.

\section{Abordagens à aprendizagem}

Biggs, Kember e Leung (2001) apresentam uma visão sistêmica das abordagens dos alunos quanto à aprendizagem, como esquematizado no modelo Presságio-Processo-Produto (3P) apresentado na Figura 1.

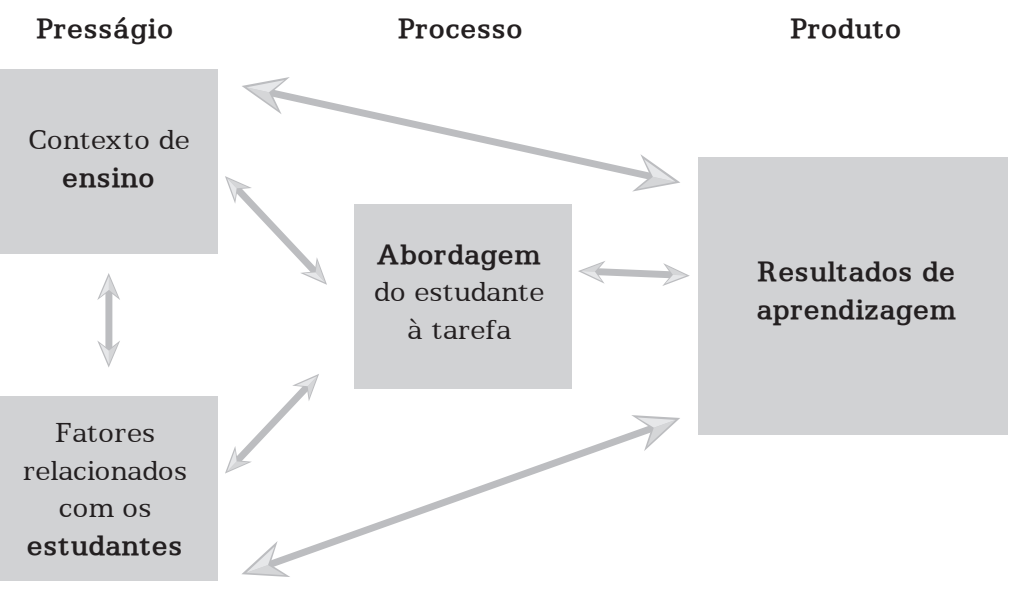

Figura 1 - Modelo 3P

Fonte: Biggs, Kember e Leung , 2001, p. 136 (adaptado).

O presságio inclui fatores relacionados com os estudantes e com o contexto de ensino que antecedem a ação. Os fatores relacionados com os estudantes dizem respeito ao conhecimento prévio, às competências e às abordagens para a aprendizagem. O contexto de ensino refere-se à natureza do conteúdo que está sendo ensinado, aos métodos de ensino e avaliação, ao clima institucional e assim por diante.

O processo diz respeito à ação do estudante e à abordagem adotada para a realização da tarefa. Os resultados da aprendizagem podem ser quantitativos, qualitativos e observáveis mediante o desenvolvimento de competências. 
No modelo apresentado, a reversibilidade das setas dá a entender que o processo ocorre nas duas direções, ou seja, os resultados podem influenciar as abordagens às tarefas e estas podem influenciar o contexto de ensino e os fatores mais diretamente relacionados com os estudantes.

Segundo Biggs (1987), o principal fator diferenciador dos resultados de aprendizagem não seria a capacidade de cognição, mas a utilização de diferentes processos de estudo consoante às "abordagens à aprendizagem". Para esse autor, tais abordagens podem ser classificadas como superficiais, profundas e estratégicas ou de alto rendimento.

A abordagem superficial diz respeito a uma postura assente no mínimo de esforço possível, ou seja, o estudante encara a aprendizagem sem o interesse de entendê-la ou aprofundá-la. Essa aprendizagem é, portanto, reprodutiva, e pauta-se pela motivação extrínseca e pelo medo do insucesso.

A abordagem profunda orienta-se pela intenção de o estudante enfrentar em profundidade a tarefa ou o conteúdo a ser aprendido. Para isso, utilizam-se as capacidades cognitivas de alto nível, como sínteses, análises, comparações e confrontações, e recorre-se ao próprio repertório cultural e cognitivo, fazendo com que esses estudantes possam atingir um nível transformador e criativo.

A abordagem estratégica ou de alto rendimento fundamenta-se na intenção de obter o máximo rendimento ou as melhores classificações por motivações intrínsecas ao sujeito.

A versão mais recente do seu questionário, o já aqui referido R-SPQ-2F, avalia apenas abordagens profundas e superficiais. O Quadro 1 apresenta as principais características dessas abordagens.

Quadro 1 - Abordagens à Aprendizagem

\begin{tabular}{|l|c|l|}
\hline \multicolumn{1}{|c|}{ Abordagem } & Motivo & \multicolumn{1}{c|}{ Estratégia } \\
\hline Superficial & Extrínseco & $\begin{array}{l}\text { Centrar-se em determinados pormenores e } \\
\text { reproduzi-los. }\end{array}$ \\
\hline Profunda & Intrínseco & $\begin{array}{l}\text { Maximizar a compreensão: ler em } \\
\text { profundidade, discutir e refletir. }\end{array}$ \\
\hline
\end{tabular}

Fonte: Biggs, 1987, p. 190 (adaptado).

Contudo, para Biggs, Kember e Leung (2001) o estudante não pode ser categorizado como "superficial" ou "profundo" com base em respostas SPQ, uma vez que essas abordagens não são determinísticas nem estáveis. As respostas SPQ variam em função das características individuais e do contexto de ensino. Dessa forma,

professor e aluno são corresponsáveis pelo resultado, o professor para estruturar as condições da formação, o aluno para envolver-se ativamente na sua concretização. Assim, uma abordagem para a aprendizagem descreve a natureza da relação entre o estudante, o contexto de ensino, a tarefa e os resultados de aprendizagem. (Biggs, Kember; Leung, 2001, p. 136). 


\section{Aprendizagem ao longo da vida}

A abordagem à temática da aprendizagem ao longo da vida remete necessariamente para uma questão de largo espectro, que a enquadra e que, simultaneamente, norteia os discursos e as práticas educativas da atualidade, em função das políticas das três principais organizações intergovernamentais com atividade nesse domínio, designadamente o Conselho da Europa, a Organização das Nações Unidas para a Educação, a Ciência e a Cultura (Unesco) e a Organização para a Cooperação e Desenvolvimento Econômico (OCDE). De fato, a problematização da aprendizagem ao longo da vida tem sido uma constante nas últimas décadas e a sua tradução discursiva e operacional, em termos de políticas educativas, tem se pautado pela diversidade e pela descontinuidade (Centeno, 2011).

O paradigma da aprendizagem ao longo da vida tem, no campo da educação, algumas idiossincrasias que se prendem com a variedade e a heterogeneidade de causas, objetivos, grupos-alvo e modelos estruturais. Essa riqueza e plasticidade, como nos tem mostrado a história das últimas décadas, constitui também a sua fraqueza, pois tal amplitude favorece múltiplas interpretações, algumas delas bastante redutoras, pondo em causa a essência que sustentou a sua gênese.

O Conselho da Europa introduziu na década de 1960 o conceito de educação permanente, entendido como um padrão de educação global, capaz de responder às necessidades dos jovens e adultos, necessidades essas cada vez mais diversificadas na educação da nova sociedade europeia. Nessa matéria, as políticas do Conselho da Europa orientaram-se pelos princípios da igualdade, da participação e da globalização.

Por seu lado, a Unesco, com base nos trabalhos desenvolvidos no âmbito das Conferências Internacionais sobre Educação de Adultos, lançou o seu programa educativo, inserido numa política suficientemente abrangente, facilitadora do estabelecimento de uma ponte com as suas atividades científicas, culturais e sociopolíticas. Os trabalhos de Faure (1972) e Lengrand (1970) estiveram na gênese de um novo humanismo, assente no pressuposto da vontade inequívoca do homem para aprender e de uma sociedade mais humana. Nesse contexto, a aprendizagem ao longo da vida tornou-se um conceito idealista e necessariamente consensual, global e flexível, por forma a que os diferentes países e respectivos regimes políticos nele se revissem. Ainda assim, autores como Lee e Friedrich (2011), reportando-se à década entre 1990 e 2000, embora reiterem o liberalismo social-democrata como ideologia dominante da Unesco, não deixam de referir a acomodação de algumas ideias neoliberais, numa sociedade dominada pelo capitalismo neoliberal.

A OCDE usou, nos anos 70, a expressão "educação recorrente", com ambições bem mais modestas e que se traduziam na oferta de oportunidades educativas menores, ao longo da vida, a serem rentabilizadas quando necessárias. Essa perspectiva encara a educação contínua como uma alternativa à educação inicial formal e revela a supremacia das preocupações de ordem econômica, das questões da produtividade e da possibilidade de 
uma relação mais flexível entre educação e formação (o lazer e a reforma foram também mencionados e submetidos à mesma lógica). Essa tendência permaneceu, mesmo quando a OCDE (OECD, 1996) passou a usar o conceito de aprendizagem ao longo da vida. Com cerca de 50 anos de existência, só em 2002 a OCDE estabeleceu um diretório para a educação, revelando o reconhecimento da importância crescente da educação em termos políticos, pela associação dinâmica do capital humano ao crescimento econômico. A educação vocacional e a formação continuam a ser recomendadas, da mesma forma que se reforçam as ligações entre empregabilidade e economia (Istance, 2011).

Enquanto os programas do Conselho da Europa e da Unesco se sustentam nos valores universais de paz, igualdade, inter-culturalidade, diversidade e direitos humanos, isto é, numa visão humanista da educação, a OCDE, pela sua natureza e também pela sua história, centrou-se nos benefícios e no potencial econômicos da educação e na sua vertente funcionalista (Centeno, 2011).

As mudanças estruturais que pautam o atual momento socioeconômico são entendidas de forma distinta pelas três organizações intergovernamentais. A Unesco sublinha a questão humanista do impacto educativo, sendo a aprendizagem ao longo da vida condição essencial para o bem-estar das pessoas e a educação uma responsabilidade coletiva. O Conselho da Europa entende as referidas mudanças como um problema político inerente aos sistemas educativos, sendo a aprendizagem ao longo da vida a ferramenta fundamental para a integração social e responsabilidade central dos diferentes Estados-membros. Por último, a OCDE perspectiva-as do ponto de vista econômico, e a implementação de políticas de aprendizagem ao longo da vida, enquanto força estabilizadora da lógica de oferta e procura, intrínseca ao mercado de trabalho, é atribuída sobretudo ao indivíduo, principal agente da sua integração socioeconômica (Centeno, 2011).

Se a Unesco sustentou a sua abordagem à educação de adultos na conceitualização de uma política de educação permanente, a OCDE formulou-a em função do conceito de educação recorrente, e o Conselho da Europa cruzou as duas opções. De fato, a perspectiva neoliberal consubstanciada nos princípios da OCDE tem vindo a contagiar o enquadramento estratégico de ação da União Europeia (Kleibrink, 2011), de tal forma que a problematização que atualmente faz da aprendizagem ao longo da vida se traduz num conceito político híbrido (Centeno, 2011).

As questões relacionadas com as qualificações profissionais, já defendidas na Estratégia de Lisboa, em 2000, continuam a nortear os esforços de cooperação em termos de educação e formação para a década de 2010 a 2020. Verificamos que as preocupações sociais, incluídas nos programas desde os anos 80 , nunca assumiram qualquer predominância, apresentando, pelo contrário, uma tendência à diluição. Essa questão é particularmente visível na definição dos indicadores e das metas estatísticas no âmbito da Estratégia de Lisboa, para objetivar e clarificar o progresso diante dos propósitos estabelecidos (por exemplo, a taxa de participação de $12,5 \%$ da população adulta em atividades de aprendizagem ao longo da vida). 
A experiência da Unesco e da União Europeia mostra que os objetivos gerais definidos pelas organizações internacionais têm sido globalmente ignorados pelos sistemas educativos dos diferentes países, mas também permite concluir que as metas estatísticas, vistas como indicadores de monitorização, não são garantias de que os objetivos são atingidos por esses mesmos sistemas educativos (Jallade, 2011). Por outro lado, o interesse da OCDE pelos benefícios sociais, concretizados no impacto da educação em termos de saúde, participação cívica e envolvimento social, são, na verdade, orientados pelos ganhos econômicos calculados. Não obstante as motivações econômicas que constituem o motor da atividade dessa organização intergovernamental, questões como a igualdade de oportunidades, a inovação, a gestão do conhecimento e o envolvimento dos adultos enquanto participantes ativos em processos de aprendizagem, assim como o reconhecimento da natureza social da aprendizagem, são indubitavelmente tópicos positivos em matéria de educação de adultos (Istance, 2011).

Concluindo, a aprendizagem ao longo da vida no contexto europeu encerra tensões e contradições, com consequências nas opções políticas, nas práticas educativas e nas prioridades teórico-científicas (Antunes, 2008). Os objetivos definidos no Programa Integrado de Aprendizagem ao Longo da Vida, de 2007 a 2013 (European..., s.d.), apresentado pela Comissão Europeia no âmbito dos programas de ação comunitária - mais tarde renomeado Programa de Aprendizagem ao Longo da Vida, de 2007 a 2013 (Decisão 2006/1720/CE do Parlamento Europeu e do Conselho Europeu) -, designadamente o aumento da empregabilidade e da coesão social e a construção de uma sociedade do conhecimento que permita o desenvolvimento econômico sustentável, a integração, a cooperação e a mobilidade dos sistemas educativos e de formação, denunciam uma postura ambivalente.

\section{Metodologia}

\section{Tipo de estudo e objetivos}

Apresentamos um estudo de natureza quantitativa, comparativocorrelacional, com caráter transversal.

Esse estudo tem como objetivos fundamentais: (i) comparar a apropriação que os estudantes fazem das diferentes formas de aprender e de estudar em função de sua percepção de desempenho acadêmico, área de formação e estatuto profissional (estudante versus trabalhador-estudante) e (ii) conhecer a relação das abordagens de aprendizagem com a idade e com a tendência/inclinação para se envolverem em atividades de ALV.

\section{Amostra}

A amostra é constituída por 163 estudantes do ensino superior politécnico, 37 do sexo masculino (22,7\%) e 126 do sexo feminino (77,3\%). 
A maior parte dos respondentes (89,6\%) frequenta o $1^{\circ}$ ciclo de estudos, que confere o grau de licenciatura; $6,7 \%$ frequentam o mestrado e $3,7 \%$ frequentam pós-graduações.

A média de idades é de 26,6 anos, com um desvio-padrão de 8,7, idade mínima de 18 e máxima de 61 anos (Gráfico 1).

Histograma

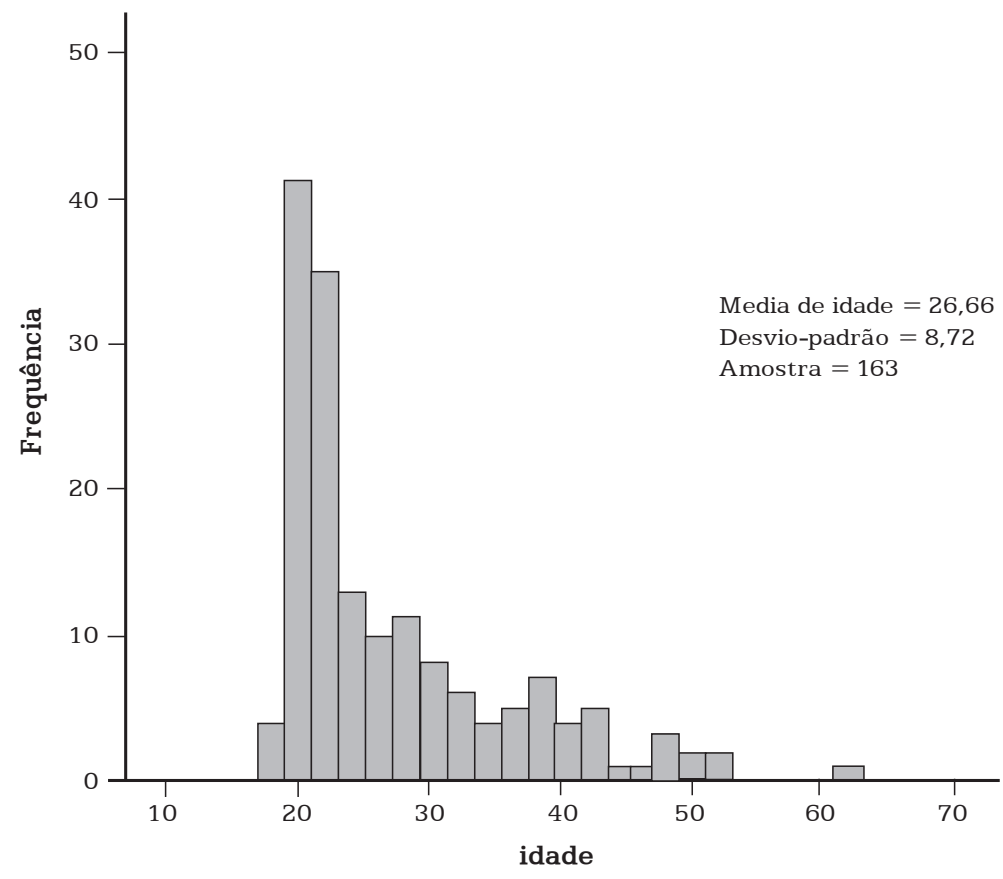

Gráfico 1 - Caracterização da Amostra em Função da Idade

Em relação à área de formação, 97 situam-se na educação (59,5\%), 62 na saúde (38\%) e 4 em outras áreas (2,5\%).

No que concerne à situação perante o trabalho, 83 são exclusivamente estudantes $(50,9 \%)$ e 77 são trabalhadores-estudantes (47,2\%). Três dos inquiridos $(1,8 \%)$ afirmam ter outra situação perante o trabalho.

A amostra foi não probabilística e, como tal, os estudantes foram selecionados por critério de conveniência, em particular pela acessibilidade facilitada, tendo-lhes sido garantido o anonimato e a confidencialidade individual dos resultados.

\section{Instrumentos de recolha de dados}

O inquérito integra três partes. A primeira centra-se em aspectos sociodemográficos, designadamente idade, sexo, nível de ensino, área de ensino (educação/saúde) e estatuto profissional. Está também incorporada nessa seção uma questão relativa à percepção dos estudantes em relação ao seu desempenho acadêmico, cuja categorização de resposta obedece à escala de classificação European Credit Transfer System (ECTS), inscrita no Sistema Europeu de Transferência de Créditos que sustenta a Declaração de Bolonha (DGES, s/d). 
Na segunda parte do inquérito, para avaliar o envolvimento dos estudantes em atividades de ALV, inspiramo-nos em alguns itens (dez) do Lifelong Learning Questionnaire, de Kirby et al. (2010). Os itens utilizados sustentam-se em características do aprendiz ao longo da vida definidas por Knapper e Cropley (2000), designadamente no estabelecimento de objetivos, na aplicação de conhecimentos e competências, na autodireção e na avaliação, na procura de informação e na adaptação de estratégias de aprendizagem. Segundo os autores, a escala avalia a tendência/inclinação dos adultos para a aprendizagem ao longo da vida, a qual resulta da combinação de traços definidos precocemente e de fatores situacionais que ocorrem posteriormente, tornando-se necessário focalizar esses traços e situações e perceber de que forma podem ser manipulados no sentido desejável. Os itens são avaliados através de escala tipo Likert de 5 pontos, desde "discordo completamente" (-2) até "concordo completamente" (+2).

Dado que não existem muitos instrumentos que permitam avaliar a aprendizagem ao longo da vida, consequência da imprecisão que pauta muitas das suas definições, não pretendemos validar para a população portuguesa a referida escala, mas tão somente recorrer a alguns dos seus itens para os relacionar com os estilos de aprendizagem utilizados pelos estudantes.

As abordagens de aprendizagem foram avaliadas na terceira parte do inquérito, por meio da tradução luso-portuguesa da validação brasileira da escala Revised Two-factor Study Process Questionnaire (R-SPQ-2F), realizada por Godoy (2009). Nessa validação para a língua portuguesa, a escala original foi, inicialmente, sujeita a processos de tradução e retrotradução. A sua versão final é constituída por 20 itens, avaliados numa escala tipo Likert de 5 pontos, desde "nunca" (1) até "sempre" (5), agrupados em quatro subescalas: motivação profunda, estratégia profunda, motivação superficial e estratégia superficial. Registra-se uma correlação linear, positiva e significativa entre motivação profunda e estratégia profunda e entre motivação superficial e estratégia superficial (moderada e baixa, respectivamente). Baixas correlações lineares, negativas e estatisticamente significativas são encontradas entre motivação profunda e superficial e entre estratégia profunda e superficial.

Os valores da consistência interna, avaliada pelo alpha de Cronbach, são compatíveis com os da escala original no que diz respeito à abordagem profunda $(\alpha=0,76)$ e até superiores no que concerne à aprendizagem superficial $(\alpha=0,74)$. A consistência interna e a validade de critério revelam as boas qualidades psicométricas do instrumento. $\mathrm{O}$ mesmo se pode dizer em relação à validade de constructo, sustentada pela análise fatorial confirmatória, realizada segundo o Modelo de Equações Estruturais (SEM). Assim, a estrutura da versão revista apresenta dois fatores não hierárquicos, distinguindo a abordagem profunda da abordagem superficial, cada uma delas constituída por dez itens. Os resultados dessa validação vão ao encontro dos resultados de análises fatoriais exploratórias e confirmatórias realizadas noutros estudos, designadamente na Espanha (Justicia et al., 2008). Um dos modelos testados por Godoy (2009) examina 
se os itens individuais se ajustam ao padrão esperado. Os índices apontam para um ajustamento mediano desse modelo ( $\mathrm{CFI}=0,79$ e RMSEA $=0,07$ ). Foi construído um segundo modelo, complementar, para avaliar a dimensionalidade, sendo que os índices de ajustamento encontrados $(\mathrm{CFI}=1,00, \mathrm{RMSEA}=0,00)$ são indicativos de um ajuste muito satisfatório em relação ao modelo original.

Perante o exposto, pareceu-nos indicada a utilização desse instrumento em língua portuguesa para avaliar as abordagens de aprendizagem em estudantes do ensino superior.

\section{Procedimentos}

Os itens do Lifelong Learning Questionnaire (Kirby et al., 2010) foram selecionados tendo em consideração os seguintes requisitos:

- dois itens por característica do aprendiz ao longo da vida, segundo Knapper e Cropley (2000), dado que as características com menos itens integram precisamente dois;

- um equilíbrio no número de itens por característica;

- a facilidade em termos do cumprimento dos equivalentes linguísticos e culturais subjacentes à tradução luso-portuguesa.

Essa tradução da escala original foi realizada por dois tradutores de inglês, que tinham o português como língua materna, para detectar imprecisões e interpretações idiossincráticas ou resultantes de influências de caráter cultural. Para identificar eventuais discrepâncias entre o sentido da tradução e os itens originais, apenas um deles conhecia os objetivos e as intenções inerentes à tradução. Posteriormente, esses mesmos itens foram disponibilizados a seis estudantes do ensino superior, para eventual identificação de problemas de compreensão. Esse pré-teste serviu para avaliar, de forma qualitativa e não estandardizada, o nível de compreensão das instruções e dos itens, com o objetivo de potenciar a obtenção de um equivalente linguístico, tão próximo quanto possível do original e, simultaneamente, de um equivalente cultural. A reflexão falada foi suscitada por meio de um fórum de sugestões e de partilha disponibilizado na plataforma de aprendizagem moodle, que permitiu equacionar a inteligibilidade e a ambiguidade dos itens, de acordo com as indicações de Bell (2010). Não foram verificadas alterações dignas de registro em relação às propostas apresentadas pelos tradutores.

As preocupações em termos de equivalência linguística e cultural sustentaram também a tradução luso-portuguesa da validação brasileira da escala Revised Two-factor Study Process Questionnaire (R-SPQ-2F), a qual foi da responsabilidade de uma tradutora com dupla nacionalidade.

Definido o questionário de recolha de dados e após autorização das diretoras das referidas escolas, foi solicitado aos estudantes o preenchimento on-line do questionário, via plataforma de aprendizagem moodle, em caráter facultativo, embora se tenha incentivado a participação. 
Aos estudantes foram garantidos o anonimato e a confidencialidade individual dos resultados.

\section{Tratamento de dados}

A análise estatística foi realizada com o Statistical Package for the Social Sciences (SPSS) - versão 19.0 -, e os procedimentos passaram pela estatística inferencial.

Recorremos a análises univariadas - Anova - na análise comparativa das abordagens de aprendizagem em função da percepção de desempenho acadêmico por parte dos estudantes. Nas comparações entre grupos de estudantes e estudantes trabalhadores, considerando a distribuição normal dos resultados e confirmada a homogeneidade de contingências dos grupos em comparação, por meio do Teste de Levene $(p>0,05)$, optamos pela estatística paramétrica, mormente ao t-test para amostras independentes.

As análises correlacionais foram realizadas com base no coeficiente de correlação linear de Pearson.

Todos os resultados foram considerados estatisticamente significativos para um intervalo de confiança de 95\% (Marôco; Bispo, 2005).

\section{Apresentação e discussão de resultados}

\section{Análises comparativas}

a) Comparação entre abordagens de aprendizagem e área de formação superior (saúde versus educação)

Não se encontram diferenças entre as áreas de formação (saúde e educação) no que diz respeito à aprendizagem como um todo e nos seus aspectos motivacionais. As diferenças entre os dois grupos de estudantes circunscrevem-se à estratégia de aprendizagem. Assim, registram-se diferenças estatisticamente significativas $(p=0,023)$ em relação à estratégia profunda $(t=-2,3)$, quando comparamos os estudantes com formação na área da educação (média =2,74) e os com formação na área da saúde $(2,92)$, com vantagem para os últimos. No que concerne à estratégia superficial, os resultados invertem-se, dando vantagem aos estudantes da área da educação (média $=3,52$ ) em relação aos da área da saúde (média=3,29, $\mathrm{t}=3,04, \mathrm{p}=0,03)$.

Esses resultados podem estar relacionados com as especificidades do contexto, assim como dos conteúdos e dos métodos de ensino e de avaliação. A reversibilidade inerente ao modelo "3P" (Biggs, 1987) prevê que o contexto de aprendizagem pode influenciar as abordagens às tarefas, designadamente na sua componente estratégica. 
b) Comparação entre abordagens de aprendizagem e percepção de desempenho acadêmico

Por meio da Anova oneway, não se verificam diferenças significativas entre a percepção de desempenho acadêmico por parte dos estudantes e as abordagens de aprendizagem, tanto para os seus aspectos motivacionais quanto para a dimensão estratégica.

O Gráfico 2 mostra a distribuição dos estudantes pelas diferentes categorias de desempenho acadêmico autopercepcionado.

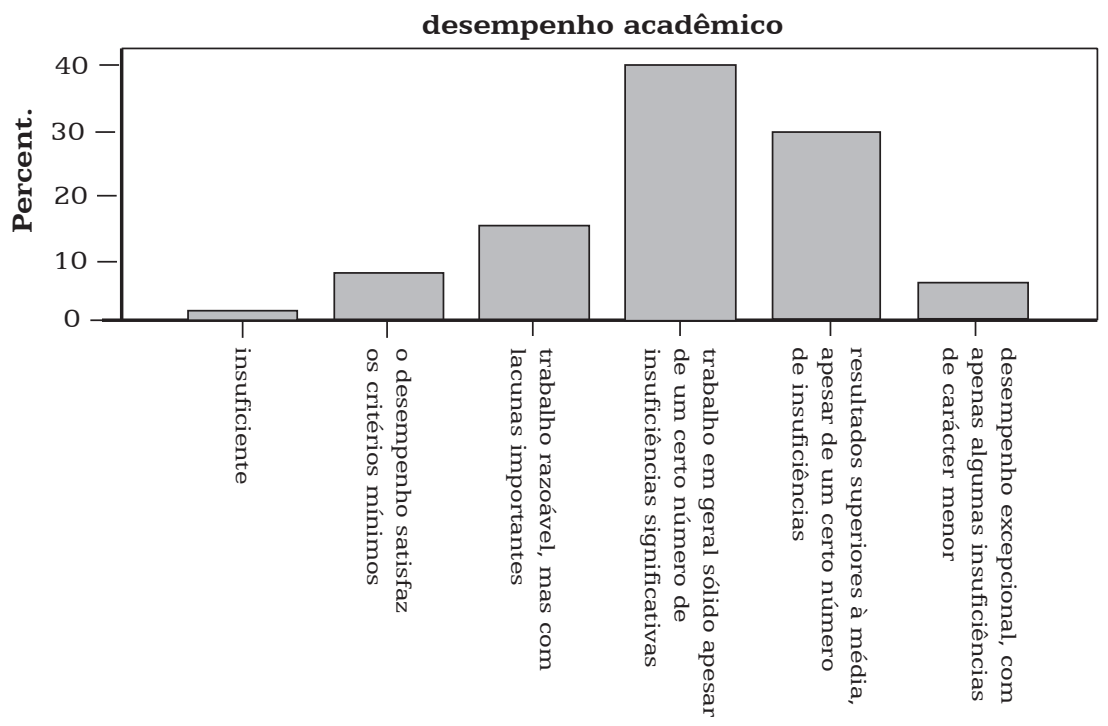

\section{Gráfico 2 - Distribuição de Frequências em Função da Percepção de Desempenho Acadêmico}

Esses resultados contrastam com os obtidos no estudo de Godoy (2009), que, avaliando o desempenho acadêmico com base nas classificações reais dos estudantes, apontam para uma correlação positiva entre motivação profunda e melhores médias em termos de notas. Também Vasconcelos, Almeida e Monteiro (2005) mostram que os estudantes que ingressam no ensino superior com melhores classificações ao nível do ensino secundário tendem a apresentar um enfoque mais compreensivo (característico da abordagem profunda) do que memorístico no estudo (característico da aprendizagem superficial), ao mesmo tempo que revelam uma autopercepção de competência e de realização acadêmica mais favorável. Assim, não parece haver um consenso em relação à articulação entre o desempenho acadêmico autopercepcionado e as abordagens de aprendizagem.

c) Comparação entre abordagens de aprendizagem e o estatuto profissional (estudante versus trabalhador-estudante)

Quando comparamos as abordagens de aprendizagem dos estudantes em função do seu estatuto profissional, verificamos apenas diferenças 
estatisticamente significativas $(\mathrm{p}=0,024)$ no tocante à estratégia profunda entre aqueles que são exclusivamente estudantes (média $=2,88$ ) e os que, em simultâneo, desenvolvem uma atividade profissional (média=2,72), $\mathrm{t}=2,28$.

Essa diferença pode ser explicada pela disponibilidade de tempo e pela necessidade de gestão deste. De fato, os estudantes que simultaneamente desempenham uma atividade profissional desenvolvem outros papéis os quais exigem competências operacionais de estruturação temporal que coadunam com as estratégias mais superficiais.

\section{Análises correlacionais}

a) Relação entre abordagens de aprendizagem e idade

O coeficiente de Pearson aponta para uma correlação negativa $(r=-0,173)$ e estatisticamente significativa $(p=0,027)$ entre a aprendizagem profunda e a idade, indicando que, à medida que a idade avança, os estudantes do ensino superior mostram-se menos propensos ao recurso a aprendizagens profundas. Não se registra uma correlação significativa entre idade e aprendizagem superficial.

Quanto à aprendizagem profunda, apenas se registra uma correlação negativa e estatisticamente significativa $(\mathrm{p}=0,044)$ em relação à estratégia profunda $(r=-0,158)$. Não se verifica qualquer correlação significativa no que diz respeito à motivação profunda.

A adoção de uma estratégia mais superficial pelos estudantes à medida que a idade avança pode, mais uma vez, relacionar-se com a multiplicidade de papéis que são desempenhados pelos estudantes com mais idade.

As exigências sociais associadas ao jovem adulto distinguem-se daquelas que se colocam na adultez madura (Erikson, 1963), com implicações em termos de investimento nas diferentes tarefas e consequente gestão temporal.

Esses resultados diferem dos apresentados por Biggs e Kirby (1983), uma vez que estes autores citam que os estudantes pré-universitários demonstram níveis mais baixos de abordagem profunda em relação aos estudantes universitários. No entanto, esse desfasamento de resultados pode justificar-se com base no contexto sociocultural e histórico dos estudantes envolvidos.

b) Relação entre abordagens de aprendizagem e a ALV

Os itens que integram a Tabela 1 estão agrupados em função das características do aprendiz ao longo da vida definidas por Knapper e Cropley (2000), designadamente:

1 - estabelecimento de metas;

2 - aplicação de conhecimento de competências;

3 - autodireção e avaliação;

4 - localização da informação;

5 - adaptação de estratégias de aprendizagem. 


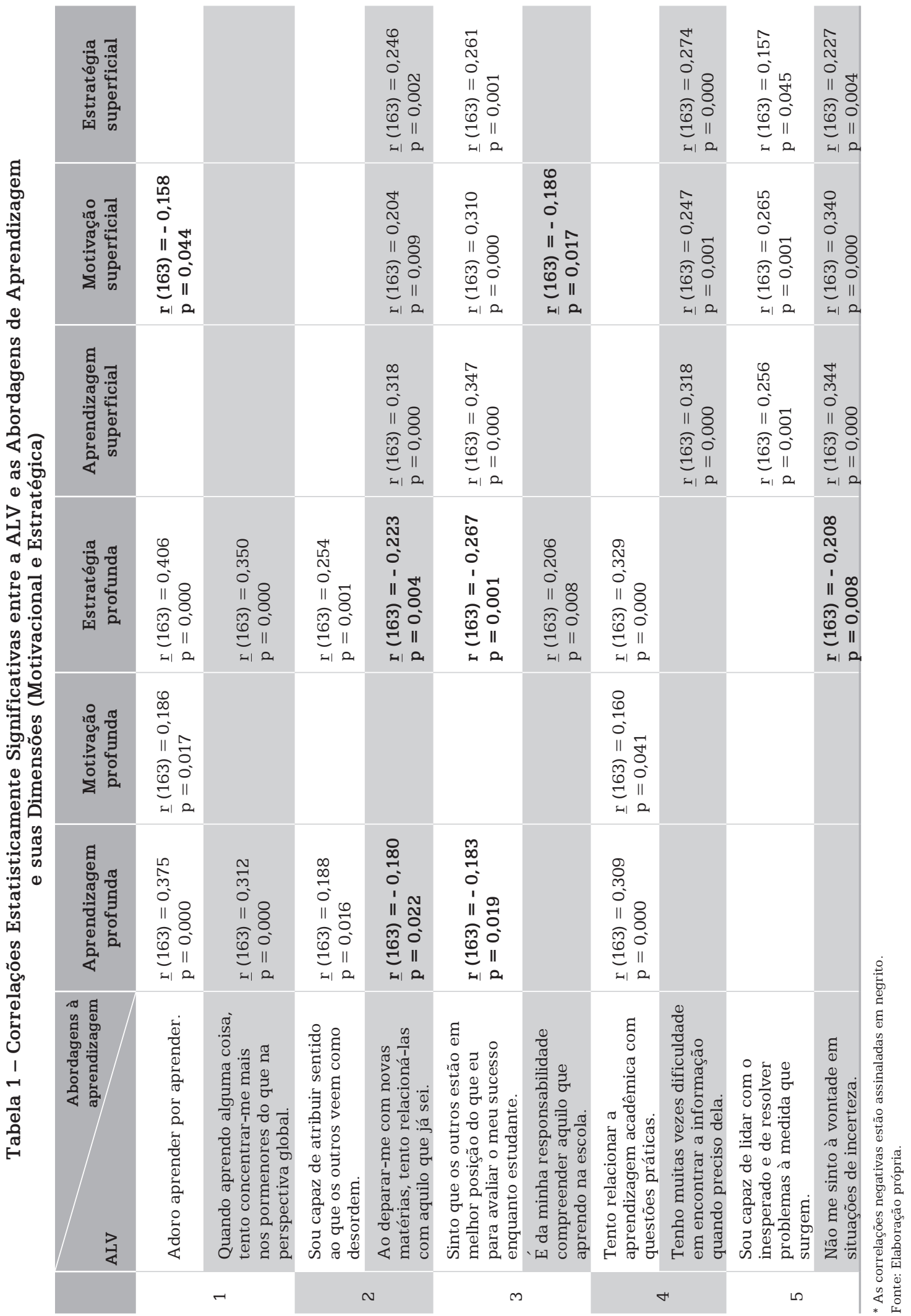


b.1) Relação entre abordagens de aprendizagem e estabelecimento de metas

Na Tabela 1, observamos uma correlação positiva entre a afirmação "adoro aprender por aprender" e a aprendizagem profunda, nas suas componentes motivacional e estratégica.

Entre os estudantes que adoram "aprender por aprender" (item 9), a aprendizagem profunda envolve não só a motivação intrínseca, dirigida à aprendizagem em si mesma, como também a exploração de processos estratégicos para concretizá-la. Caso o estudante não identifique reforços extrínsecos, também não tem essa percepção, o que justifica a sua correlação negativa com a estratégia superficial.

Por outro lado, para esses estudantes que apresentam uma aprendizagem profunda, a centração nos pormenores (item 14) não invalida a existência de uma visão macroscópica do conteúdo da aprendizagem. Implica, em todo caso, uma estratégia mais minuciosa e, portanto, mais aprofundada na análise do conteúdo, traduzida na correlação positiva com a estratégia profunda. Valadares e Moreira (2009) corroboram com essa ideia quando articulam os processos de diferenciação progressiva e reconciliação integradora ou integrativa com a aprendizagem significativa.

b.2) Relação entre abordagens de aprendizagem e aplicação de conhecimentos e competências

A atribuição de significado, avaliada pelo item 5, implica articulação e coerência entre as diversas informações e conhecimentos que sustentam a aprendizagem profunda. Essa articulação só é possível com o recurso a estratégias profundas.

A correlação negativa entre a articulação de novas matérias a aprender com os conhecimentos anteriores (item 12) e a motivação profunda, aliada à correlação positiva com a motivação e a estratégia superficiais, podem ser explicadas pelo entendimento que os estudantes têm desse construto. De fato, é possível que os estudantes que apresentam uma motivação profunda entendam o relacionamento com as matérias anteriores adquiridas como uma limitação à exploração de novos conteúdos. Em contraposição, esse relacionamento pode ser visto como uma estratégia de aprendizagem a rentabilizar, sobretudo quando o investimento na tarefa de aprendizagem é reduzido e quando o estudante está mais centrado nos resultados do que nos processos de aprendizagem e na aprendizagem em si mesma.

b.3) Relação entre abordagens de aprendizagem e autodireção e avaliação

A avaliação da aprendizagem pode ser conjugada com questões de autonomia, autodireção e autorregulação na aprendizagem. A correlação positiva encontrada entre o privilegiar da heteroavaliação e a aprendizagem superficial, tanto do ponto de vista motivacional como do estratégico, implica atribuições externas. 
Os estudantes, ao delegarem aos outros a responsabilidade da avaliação (item 8) do sucesso na aprendizagem, podem, em caso de insucesso, construir mecanismos defensivos de proteção do "eu" (Fontaine, 1990). Para os estudantes com uma abordagem profunda à aprendizagem, a questão da avaliação só se coloca em nível estratégico, tal como nos revela a correlação negativa observada. As atribuições causais (internas ou externas) justificam não apenas a avaliação da aprendizagem como a responsabilização por ela (item 13).

A esse respeito, Biggs, Kember e Leung (2001) mencionam que os métodos de ensino e de avaliação frequentemente encorajam a abordagem superficial quando não estão devidamente alinhados com os objetivos de ensino.

b.4) Relação entre abordagens de aprendizagem e localização da informação

Na Tabela 1, observamos uma correlação positiva entre a abordagem profunda (motivação e estratégia) e a afirmação "tento relacionar a aprendizagem acadêmica com questões práticas". Por outro lado, encontramos uma correlação negativa entre a abordagem superficial (motivação e estratégia) e a afirmação "tenho muitas vezes dificuldade em encontrar a informação quando preciso dela".

Os estudantes que investem de forma aprofundada nas aprendizagens transferem,

articuladamente, as estratégias de pensamento utilizadas em um dado contexto, bem como os conhecimentos gerados a partir delas, para outros. A transferência é a base da acumulação do conhecimento e da aprendizagem humana, marcando em especial a possibilidade de, partindo do conhecido (conteúdos, estratégias, habilidades, etc.), articulá-lo de outra forma, chegando a novas soluções, conclusões e ideias (Davis; Nunes; Nunes, 2005, p. 210).

Daí que relacionem a aprendizagem acadêmica com questões práticas (item 10). Essa preocupação exige não apenas uma motivação intrínseca na aprendizagem como também estratégias que facilitem a referida relação.

O investimento profundo nas aprendizagens faz com que se desenvolvam competências transversais, mormente as capacidades de seleção de informação, análise e síntese. O desenvolvimento dessas competências facilita a busca da informação que sustenta o processo de aprendizagem. Assim, compreende-se que os estudantes com uma abordagem superficial (tanto do ponto de vista motivacional como do estratégico), ao não terem oportunidade de desenvolver essas competências, tenham uma percepção de dificuldades em encontrar informação quando dela necessitam (item 11).

b.5) Relação entre abordagens de aprendizagem e adaptação de estratégias de aprendizagem

A correlação positiva entre a abordagem superficial e a afirmação "sou capaz de lidar com o inesperado e de resolver problemas à medida 
que surgem" pode explicar-se pelo fato de que na abordagem superficial o investimento do estudante é menor.

Nesse contexto, percebe-se que se constrói a autopercepção de capacidade para lidar com o imprevisto (item 3), mesmo que o resultado da sua ação seja discutível ou até inapropriado. Efetivamente, ao centrar-se nos resultados da aprendizagem, as questões processuais e metodológicas, que garantem segurança na ação, acabam por não serem tidas como muito importantes.

No que diz repeito ao item 4 - o desconforto frente a situações de incerteza -, este está correlacionado positivamente com a aprendizagem superficial e negativamente com a estratégia profunda.

Se na abordagem profunda a incerteza pode constituir-se como desafio à adoção de estratégias de aprendizagem profunda, na abordagem superficial é frequente os estudantes depararem-se com situações nas quais não têm certezas em relação à eficácia de seus conhecimentos e suas competências para a resolução.

$\mathrm{O}$ "sentir-se à vontade com lidar com a incerteza" está diretamente relacionado aos processos metacognitivos, tais como o processo de tomada de decisão em situações de elevada complexidade que são comuns na atualidade, em que o ambiente é muito dinâmico, ou seja, encontra-se em constante mudança (Rosenhead; Mingers, 2001).

\section{Considerações finais}

Com a intenção de analisar as diferentes abordagens à aprendizagem de estudantes do ensino superior e a sua disposição para o envolvimento em atividades de aprendizagem ao longo da vida, procedemos a este estudo comparativo e correlacional.

Mais especificamente, este estudo teve como objetivos: (i) comparar a apropriação que os estudantes fazem das diferentes formas de aprender e de estudar em função de sua percepção de desempenho acadêmico, sua área de formação e seu estatuto profissional (estudante versus trabalhadorestudante); e (ii) conhecer a relação das abordagens de aprendizagem com a idade e com a tendência/inclinação para se envolverem em atividades de aprendizagem ao longo da vida.

Relativamente à comparação entre as abordagens à aprendizagem e as características dos estudantes, verificamos que as principais diferenças, quando constatadas, situam-se no nível das estratégias utilizadas. Enquanto as abordagens não se distinguem em função da percepção do desempenho acadêmico, a dimensão estratégica diferencia-se de acordo com a área de formação e o estatuto profissional do estudante.

No que diz respeito à relação entre as abordagens de aprendizagem e a idade, no nosso estudo verificou-se que os estudantes mais velhos tendem a recorrer a abordagens mais superficiais, particularmente na 
sua componente estratégica. Parece-nos, conforme referimos, que esse resultado pode explicar-se com as demandas do quotidiano e a assunção de tarefas em simultâneo para as quais são exigidas soluções rápidas e eficazes.

Há ainda outro importante fator a ponderar, tal como referem Biggs, Kember e Leung (2001): as estratégias de ensino e os métodos de avaliação podem impelir os estudantes a optarem por estratégias inscritas numa abordagem superficial.

Quanto à relação entre as abordagens de aprendizagem e a tendência/ inclinação para os estudantes se envolverem em atividades de aprendizagem ao longo da vida, esta foi agrupada por meio das características do aprendiz ao longo da vida, nomeadamente: estabelecimento de metas; aplicação de conhecimento de competências; autodireção e avaliação; localização da informação; e adaptação de estratégias de aprendizagem.

Quanto ao estabelecimento de metas, o gosto da aprendizagem pela aprendizagem, correlacionado positivamente com a aprendizagem profunda, relaciona-se com a motivação intrínseca e com o valor afetivo que o sujeito imputa à aprendizagem em si mesma. Por sua vez, a capacidade de articular uma visão macroscópica dos conteúdos de aprendizagem com os pormenores que uma aprendizagem profunda exige são reveladores de competências operacionais que ultrapassam a instrumentalidade do saber-fazer.

No que diz respeito à aplicação de conhecimentos e competências, observamos uma tendência para a abordagem superficial ao se "rentabilizarem" os conhecimentos e as competências já desenvolvidas na resolução de novos desafios.

No que concerne à autodireção e avaliação, a opção por abordagens mais superficiais está relacionada com a responsabilização do outro. No caso dos estudantes que investem em aprendizagens profundas, há um maior controle individual, que se traduz numa aprendizagem autorregulada. Essa aprendizagem implica processos mentais e estratégias em que os estudantes se envolvem deliberadamente, para melhorar as suas aprendizagens e performances. A autocompetência e autonomia percebidas, a orientação para a tarefa e o clima de aprendizagem na sala de aula interferem na motivação intrínseca.

A localização da informação é uma competência que depende da sua acessibilidade/seleção, assim como da capacidade de relacionar a aprendizagem com questões práticas. Verificamos que os estudantes que tendencialmente apresentam uma abordagem superficial têm mais dificuldades em encontrar a informação. Essa é uma competência transversal, tal como a aplicação dos conhecimentos na resolução de problemas práticos, que no seu conjunto constitui "um denominador comum presente quer nos saberes adquiridos para lá das disciplinas e áreas quer nas atividades cognitivas de aprendizagem" (Pacheco, 2011, p. 49).

Finalmente, em relação à adaptação de estratégias de aprendizagem, constatamos que os estudantes que tendem a adotar uma abordagem 
superficial julgam-se mais capazes de lidar com o inesperado, embora se sintam desconfortáveis em situações de incerteza, contrariamente ao verificado na dimensão estratégica da aprendizagem profunda.

Estudar as relações entre as abordagens à aprendizagem e algumas características do aprendiz ao longo da vida permitiu-nos equacionar possíveis implicações práticas para os contextos formais de ensino superior, que passamos a mencionar:

- Considerando que as aprendizagens superficial e profunda não são mutuamente exclusivas nem determinísticas, cabe aos agentes educativos promoverem um contexto facilitador de aprendizagens significativas, características de uma abordagem mais profunda. Nesse sentido, Biggs, Kember e Leung (2001) citam que a presença de uma abordagem mais superficial pode ser um indicador de que algo não está a correr bem nos métodos de ensino e de avaliação privilegiados pelos professores.

- O estímulo ao estabelecimento de relações e de ressignificações dos conteúdos, próprios das aprendizagens profundas, é fundamental para a realização de transferências. É a transferência que possibilita "estender habilidades e posturas deliberadamente para outros cenários, ajudando os alunos a pensar sobre suas ideias, articulando-as com as veiculadas em outras disciplinas e aplicando-as a contextos escolares e não escolares" (Davis; Nunes; Nunes, 2005, p. 210), onde se inserem as atividades de aprendizagem ao longo da vida.

- O "gosto de aprender por aprender" não é necessariamente uma condição determinada a priori. O modelo cíclico dos "3P" ilustra a interdependência de todos os elementos constitutivos do processo de ensino e aprendizagem. Dessa forma, o professor, por meio da promoção de um clima propício à aprendizagem, tem a possibilidade de, progressivamente, suscitar esse gosto e estimular os processos autônomos de descoberta, característicos de uma aprendizagem autorregulada.

- Essa plasticidade trabalhada em contexto formal no ensino superior poderá refletir-se na disposição que os estudantes apresentam para se envolver em atividades ao longo da vida. Desse ponto de vista, educar os estudantes do ensino superior e orientá-los para abordagens profundas pode constituir-se num contributo importante nesse propósito.

- Diante dos desafios que se impõem na sociedade atual, as aprendizagens ao longo da vida tornam-se um imperativo. Como tal,

seria preciso ensinar estratégias que permitiriam enfrentar os imprevistos, o inesperado e a incerteza, e modificar seu desenvolvimento, em virtude das informações adquiridas ao longo do tempo. E preciso aprender a navegar em um oceano de incerteza em meio a arquipélagos de certeza (Morin, 2001, p. 14). 


\section{Referências bibliográficas}

ANTUNES, F. A nova ordem educacional: espaço europeu de educação e aprendizagem ao longo da vida. Coimbra: Almedina, 2008.

BELL, J. Como realizar um projecto de investigação. 5. ed. Lisboa: Gradiva, 2010.

BIGGS, J.; KEMBER, D.; LEUNG, D. The revise two-factor study process questionnaire: R-SPQ-2F. British Journal of Education Psychology, Chichester, v. 71, p. 133-149, 2001.

BIGGS, J. From theory to practice: a cognitive systems approach. Higher Education Research and Development, Milperra, n. 12, p. 73-86, 2001.

BIGGS, J. Student approaches to learning and studying. Hawthorn: Australian Council for Educational Research, 1987.

BIGGS, J. What do inventories of students' learning process really measure? A theoretical review and clarification. British Journal of Education Psychology, Chichester, v. 83, p. 3-19, 1993.

BIGGS, J.; KIRBY, J. Approaches to learning in universities and CAEs. Vestes, Melbourne, v. 27, n. 2, p. 3-9, 1983.

CENTENO, V. Lifelong learning: a policy with a long past but a short history. International Journal of Lifelong Education, London, v. 30, n. 2, p. 133-150, 2011.

DAVIS, C.; NUNES, M.; NUNES, C. Metacognição e sucesso escolar: articulando teoria e prática. Cadernos de Pesquisa, São Paulo, v. 35, n. 125, p. 205-230, 2005.

PORTUGAL. Ministério da Ciência, Tecnologia e Ensino Superior (MCTES). Direção-Geral de Ensino Superior (DGES). ECTS: European Credit Transfer System [Sistema europeu de transferência de créditos]. Lisboa: DGES, [s.d.]. Disponível em: <http://tinyurl.com/bfbkqpv. >. Acesso em: 26 jan. 2013.

ENTWISTLE, N. Taking stock: an overview of key research findings. In: HUGHES, J.; MIGHTY, J. Taking Stock: research on teaching and learning in higher education. Montreal; Kingston: McGill-Queens University Press, 2010. (Queen's Policy Studies Series). p. 15-57.

ENTWISTLE, N.; RAMSDEN, P. Understanding student learning. London: Croom Helm, 1983. 
ERIKSON, E. Infância e sociedade. Rio de Janeiro: Zahar, 1963.

EUROPEAN COMISSION EDUCATION \& TRAINING. The lifelong learning programme: education and training opportunities for all. Disponível em: <http://ec.europa.eu/education/lifelong-learningprogramme/doc78_en.htm>. Acesso em: 12 jan. 2009.

FAURE, E. et al. Learning to be: the world of education today and tomorrow. Paris: UNESCO, 1972.

FONTAINE, A. M. Motivação e realização escolar. In: CAMPOS, B. Psicologia do desenvolvimento e da educação de jovens. Lisboa: Universidade Aberta, 1990. p. 93-132.

GARRISON, D. Self-directed learning: toward a comprehensive model. Adult Education Quarterly, Califórnia, v. 48, n. 1, p. 18-33, 1997.

GODOY, S. Validação para o Brasil da escala Revised two-factor Study Process Questionnaire (R-SPQ-2F). 2009. 112 f. Tese (Doutorado em Ciências) - Escola de Enfermagem de Ribeirão Preto, Universidade de São Paulo, Ribeirão Preto, 2009.

ISTANCE, D. Education at OECD: recent themes and recommendations. European Journal of Education, Chichester, v. 46, n. 1, p. 87-100, 2011.

JALLADE, J-P. International approaches to education: a review of some major cooperative programmes. European Journal of Education, Chichester, v. 46, n. 1, p. 7-24, 2011.

JARVIS, P. Inquiry Journal of Lifelong Education. International Journal of Lifelong Education, London, v. 29, n. 4, p. 397-400, 2010.

JUSTICIA, F. et al. The revised two factor study process questionnaire (R-SPQ-2F): exploratory and confirmatory factor analyses at item level. European Journal of Psychology of Education, Chichester, v. 23, n. 3, p. 355-372, 2008.

KIRBY, J. et al. Development of a scale to measure lifelong education. International Journal of Lifelong Education, London, v. 29, n. 3, p. 291302, 2010 .

KNAPPER, C. K.; CROPLEY, A. Lifelong learning in higher education. London: Kogan Page, 2000.

KLEIBRINK, A. The EU as a norm entrepreneur: the case of lifelong learning. European Journal of Education, Chichester, v. 46, n. 1, p. 70-84, 2011. 
LEE, M.; FRIEDRICH, T. Continuously reaffirmed, subtly accomodated, obviously missing and fallaciously critiqued: ideologies in UNESCO's lifelong learning policy. International Journal of Lifelong Education, London, v. 30, n. 2, p. 151-169, 2011.

LENGRAND, P. An introduction to lifelong education. Paris: UNESCO, 1970.

MAROCO, J.; BISPO, R. Estatística aplicada às ciências sociais e humanas. Lisboa: Climepsi, 2005.

MORIN, E. Os sete saberes necessários à educação do futuro. São Paulo: Cortez, 2001.

ORGANIZATION FOR ECONOMIC CO-OPERATION AND DEVELOPMENT (OECD). Lifelong learning for all. Paris: OECD, 1996.

OLESEN, H. Lifelong Learning: a challenge for adult education research. In: CASTRO, R.; SANCHO, A.; GUIMARÃES, P. Adult education: new routes in a new landscape. Braga: Unidade de Educação de Adultos/ Universidade do Minho, 2006.

PACHECO, J. Discursos e lugares das competências em contexto de educação e formação. Porto: Porto Editora, 2011.

PIRES, A. Reconhecimento e validação das aprendizagens experienciais. Sísifo: revista de ciências da educação, Lisboa, n. 2, p. 5-19, 2007.

PREECE, J. Research in adult education and lifelong learning in the era of CONFINTEA VI. International Journal of Lifelong Education, London, v. 30, n. 1, p. 99-117, 2011.

ROSENHEAD, J.; MINGERS, J. Rational analysis for a problematic world: problem structuring methods for complexity, uncertainty and conflit. West Sussex: John Willey \& Sons, 2001.

SANTOS, P.; MAIA, J. Análise factorial confirmatória e validação preliminar de uma versão portuguesa da escala de autoestima de Rosenberg. Psicologia, teoria, investigação e prática, Braga, n. 2, p. 253268, 2003.

UNESCO. Relatório global sobre aprendizagem e educação de adultos. Brasília, DF: UNESCO, 2010.

CONFERÊNCIA INTERNACIONAL DE EDUCAÇÃO DE ADULTOS, 6., 2009, Belém. Anais eletrônicos... Belém: UNESCO, 2009. Disponível em: <http://www.unesco.org/pt/confinteavi/confintea-vi/objectives/>. Acesso em: 10 jan. 2009. 
VASCONCELOS, R.; ALMEIDA, L.; MONTEIRO, S. Métodos de estudo em alunos do $1^{\circ}$ ano da universidade: métodos de estudo na universidade.

Psicologia Escolar e Educacional, Maringá, v. 9, n. 2, p. 195-202, 2005.

VALADARES, J.; MOREIRA, M. A teoria da aprendizagem significativa: sua fundamentação e implementação. Coimbra: Almedina, 2009.

YOUNG, M. The motivational effects of the classroom environment in facilitating self-regulated learning. Journal of Marketing Education, Califórnia, n. 27, p. 25-40, 2005.

Rita Manuela A. Barros, doutora em Ciências da Educação pela Universidade de Santiago de Compostela, é professora adjunta na Escola Superior de Educação Jean Piaget, em Vila Nova de Gaia, Portugal.

rbarros@gaia.ipiaget.org

Angélica Maria Reis Monteiro, doutora em Ciências da Educação pela Universidade do Porto, é diretora adjunta na Escola Superior de Educação Jean Piaget, em Vila Nova de Gaia, Portugal.

amonteiro@gaia.ipiaget.org

José António Marques Moreira, doutor em Ciências da Educação pela Universidade de Coimbra, é professor auxiliar no Departamento de Educação e Ensino a Distância na Universidade Aberta, em Lisboa, Portugal. jmoreira@uab.pt

Recebido em 06 de agosto de 2013.

Aprovado em 15 de abril de 2014. 